\title{
Reciclaje de botellas de PET para obtener fibra de poliéster
}

\author{
Laura Mansilla Pérez, Marcos Ruiz Ruiz \\ Ingeniería Industrial n. 27, 2009, ISSN 1025-9929, pp. 123-137
}

REsumen: Las botellas de tereftalato de polietileno (PET) pueden ser recuperadas y recicladas, con el fin de obtener nuevos productos. Dado que los envases de bebidas gaseosas son elaborados mayoritariamente de este material, en las siguientes líneas presentaremos una visión general del mercado de este sector en Lima y de su evolución en los últimos años. El proceso de reciclaje, que parte de la adecuada selección de las botellas para ser molidas, es mecánico y relativamente sencillo. Las hojuelas (flakes) de PET obtenidas se transforman en fibra corta de poliéster. Este insumo, combinado con otras fibras en proporciones pertinentes, puede usarse para la fabricación de ropa, relleno de cojines, alfombras, cortinas, etcétera. Además, reciclar el PET contribuye a cuidar nuestro medio ambiente.

Palabras clave: tereftalato de polietileno / fibra corta de poliéster / botellas de bebidas gaseosas / reciclaje

\section{Recycling PET bottles to obtain polyester staple fibre}

ABSTRACT: Polyethylene terephthalate (PET) bottles can be recycled and used to manufacture new products in different industrial areas. Soda bottles are made mainly of PET. Below, we give a general overview of the soda drink market in Lima (Peru), as well as how it has evolved in the last few years. Furthermore, we explain a simple mechanical process to produce staple fibre. The process begins by shredding the bottles into small fragments called flakes. PET flakes are used as raw material for a wide variety of products that would otherwise be made of polyester. Examples include polyester staple fibre, a base material for the production of clothing, pillows or carpets. Recycling PET bottles can help save our planet by taking care of our environment.

Keywords: polyethylene therephthalate (PET) / staple fibre / soda drink bottles / recycling 


\section{INTRODUCCIÓN. LA CONCIENCIA AMBIENTAL Y EL RECICLAJE}

La conciencia ambiental, que lleva a la ingeniería a la configuración de sistemas tecnológicos y procesos productivos eficientes que armonicen con el entorno, deriva necesariamente en la idea de reciclar. El desarrollo industrial y tecnológico, si bien ha traído innumerables beneficios sociales, también ha tenido repercusiones negativas y de difícil reversibilidad en el planeta. Por ello, resulta un imperativo la formación en una sólida ética ambiental en las facultades y escuelas de ingeniería, así como la divulgación sostenida de alternativas de tecnologías limpias que contribuyan a palear los efectos nocivos que la sociedad posmoderna está generando.

En tal sentido, y dado el importante auge que ha tenido la industria de bebidas gaseosas en los últimos años, en el mundo se han desarrollado diferentes tecnologías que recuperan y reutilizan los envases plásticos de bebidas gaseosas no retornables y retornables, ${ }^{1}$ haciendo de este sector un poco menos dañino para el entorno ambiental.

Las botellas de PET, ${ }^{2}$ polímero producto de la policondensación del ácido tereftálico y el glicol etilénico, han ido desplazando en el tiempo a las botellas de vidrio y constituyen la forma clásica y más extendida de presentar al mercado actual los refrescos y bebidas gaseosas, entre otros productos. El PET se desarrolló inicialmente en la década de 1940, y pese a que al comienzo se empleaba para la producción de una fibra para la industria textil que combinaba muy bien con otras fibras, su uso se extendió a la fabricación de cintas de empaque en la década de 1960 y, posteriormente, en los años setenta, a la manufactura de los mencionados envases.

En las siguientes líneas abordaremos brevemente la dinámica de importación y consumo de las resinas de PET y la evolución del sector industrial de bebidas gaseosas en nuestro país, para luego proponer una alternativa de proceso de reciclaje mecánico orientado a la producción de fibra corta de poliéster. Sin embargo, resulta pertinente hacer la salvedad respecto de las infinitas posibilidades de recuperación de PET existentes, que no son materia de análisis más profundo en este artícu-

1 Recordemos que un envase con un ciclo de vida útil, por muy extenso que este sea, termina indefectiblemente también en el botadero.

2 Tereftalato de polietileno. 
lo. Algunas de ellas corresponden a reciclajes químicos, en los que gracias a tratamientos diversos como alcohólisis, glicólisis, hidrólisis, saponificación, etcétera, se efectúa la reconversión del polímero a los monómeros de partida o sus componentes base. En otros métodos de reciclaje energéticos, el calor de los residuos de PET incinerados se recupera en una caldera para uso de vapor o generación de energía eléctrica. Esta última alternativa, sin embargo, puede traer consigo emisiones tóxicas si no se maneja adecuadamente el polímero incinerado.

\section{LA NATURALEZA DEL PET Y LA DINÁMICA DE LA IMPORTACIÓN DE RESINAS PET EN EL PAÍS}

Un kilogramo de PET está compuesto por $64 \%$ de petróleo, $23 \%$ de derivados líquidos de gas natural y $13 \%$ de aire. ${ }^{3}$ El paraxileno, extraído del petróleo crudo, permite la obtención del ácido tereftálico al oxidarse con el aire. Por su parte, el etileno, derivado del gas natural, se oxida con aire para la obtención del etilenglicol. El PET resulta de la combinación del ácido tereftálico y el etilenglicol.

Entre los usos más generalizados de este polímero destaca la fabricación de preformas de botellas para la industria de bebidas gaseosas y agua mineral, así como para cosméticos, medicinas, aceites y frascos de todo tipo. También se fabrican cintas de video y audio, bandejas para microondas, geotextiles y fibras para la industria textil.

En cuanto al PET, usado masivamente en la industria de bebidas, registra una participación de alrededor del 65\% de todos los envases. Este insumo se importa como resina ante el escaso desarrollo de la industria petroquímica nacional y su costo incide considerablemente en los márgenes industriales. Ello hace que la industria de bebidas gaseosas sea muy dependiente de las fluctuaciones en la cotización internacional del insumo. En el año 2007 el precio promedio de importación de resina PET alcanzó los US\$1.471 por TM. La principal procedencia de la resina para la elaboración de envases es Estados Unidos (43\%) y Taiwán (41\%). ${ }^{4}$ Otros países proveedores son China, Korea, Hong Kong y Colombia.

3 Plastivida Argentina. Entidad técnico profesional especializada en plásticos y medio ambiente. "Características y usos de los plásticos: El tereftalato de polietileno" [en línea]. Créditos <http://www.plastivida.com.ar>. [Consulta: 20 de abril del 2009.]

4 Maximixe. CASER, Club de Análisis de Riesgos. Gaseosas. Marzo del 2008, p. 26. 
A continuación, el gráfico 1 muestra tal distribución de proveedores, y el gráfico 2 presenta el consumo de resinas de PET en los últimos años.

\section{Gráfico 1}

Proveedores de resinas PET (\% de participación)

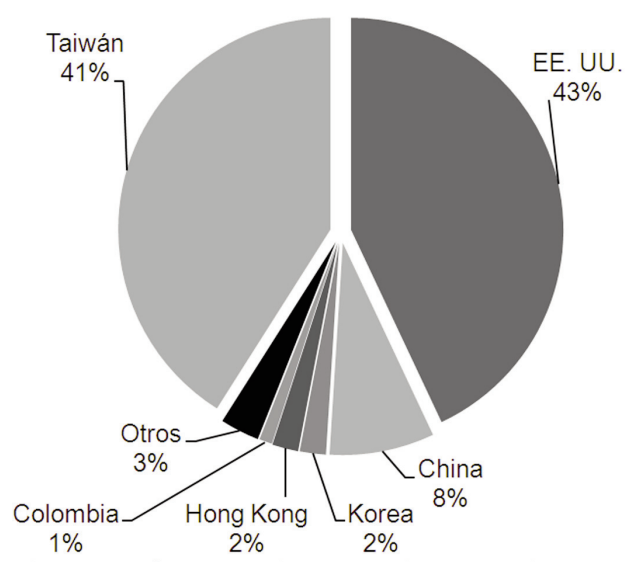

Fuente: Sunat / Maximixe.

\section{Gráfico 2}

Consumo de resinas PET (miles de TM)

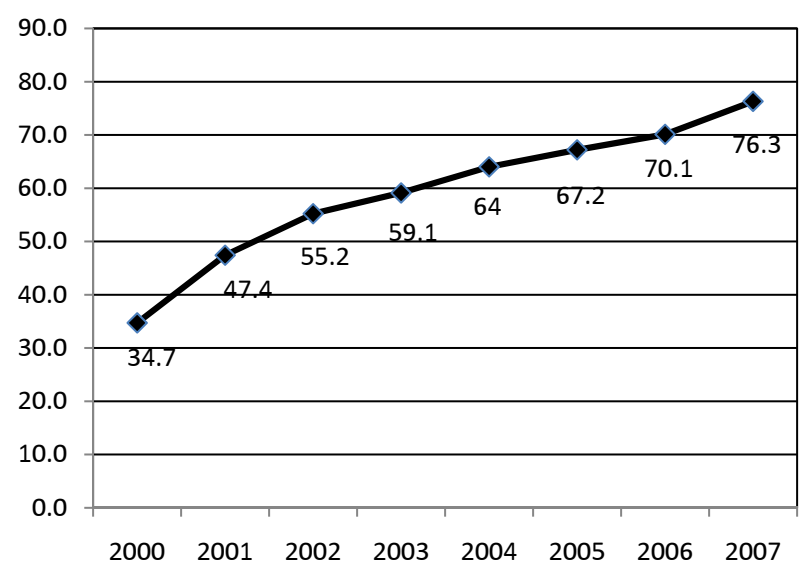

Fuente: Ministerio de la Producción (Produce) / Instituto Cuánto. 
Los últimos años se caracterizaron por una atractiva demanda interna, que benefició a la industria de bebidas gaseosas. Este escenario favoreció, como era de esperar, la entrada de nuevas marcas al mercado que generaron un mayor aumento en las importaciones de resinas PET para la fabricación de las preformas de botellas. Son dos las empresas locales que fabrican en conjunto más de 1.300 millones de envases PET al año. Se trata de San Miguel Industrial y Amcor Pet Packaging, cuya producción presenta cierta estacionalidad a causa del fuerte aumento de la demanda de sus compradores durante los meses de verano. En los meses invernales procuran compensar la caída en la demanda (especialmente de botellas familiares) con envíos al exterior y con la fabricación de envases personales. ${ }^{5}$ En el 2008, San Miguel Industrial destinó el 83\% de sus exportaciones (alrededor de 400 millones de unidades de preformas PET) a Colombia, Bolivia y Ecuador. Por su parte, Amcor Pet Packaging destinó el 47\% de sus exportaciones (89 millones de preformas PET) a Venezuela y República Dominicana. El mercado colombiano se está volviendo atractivo para esta empresa, ya que los volúmenes de exportación a ese país van en aumento. ${ }^{6}$ El gráfico 3 y el gráfico 4 presentan los principales importadores de resinas PET para la fabricación de preformas y el precio promedio de tales importaciones, respectivamente.

\section{Gráfico 3}

Principales importadores de resinas PET

(\% de volumen de importación en el 2007)

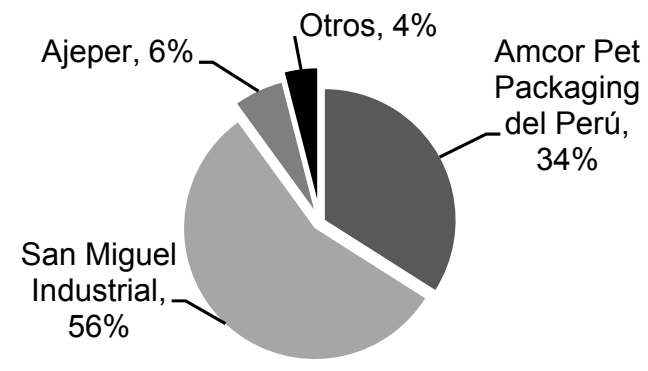

Fuente: Sunat / Maximixe.

5 Maximixe. CASER, Club de Análisis de Riesgos. Envases plásticos. Febrero, 2009, p. 82. 6 Ibídem, p. 139. 


\section{Gráfico 4}

Precio promedio de importación de resinas PET (US\$/TM)

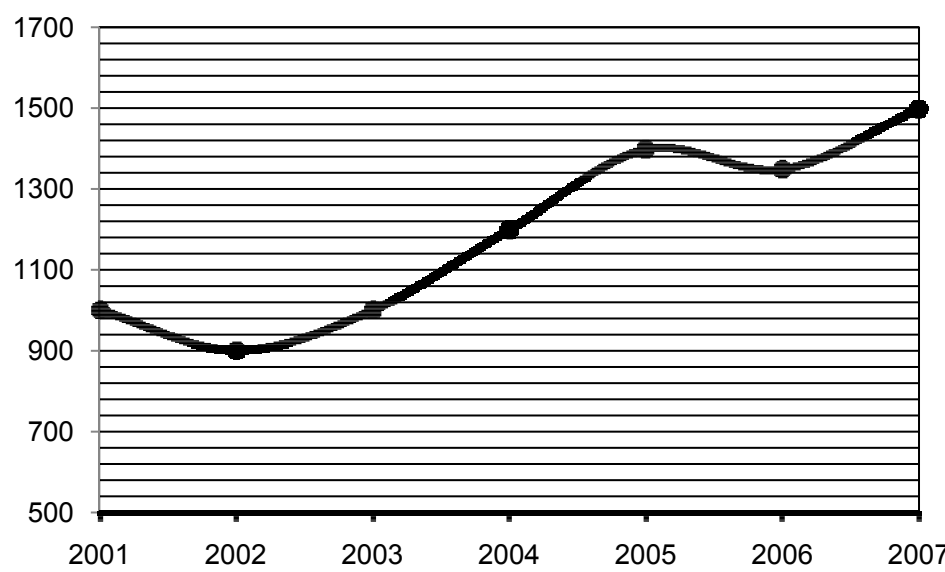

Fuente: Ministerio de la Producción (Produce) / Maximixe.

\section{LA DINÁMICA LOCAL EN LA PRODUCCIÓN DE BEBIDAS GASEOSAS}

En la última década, el crecimiento de este sector industrial se ha presentado prácticamente de manera sostenida, aunque con algunas desaceleraciones estacionarias. El dinamismo reciente de bienes sustitutos como néctares, jugos de frutas y el agua embotellada puede considerarse una de las causas de estas desaceleraciones, así como una leve tendencia del consumidor a adquirir productos naturales y saludables.

En el año 2007, por ejemplo, el sector presentó una desaceleración al registrar un discreto crecimiento de $0,3 \%$ frente al 4,9\% del 2006. Si bien la prolongación del invierno del 2007 pudo haber afectado la demanda de bebidas gaseosas, también lo han hecho los altos precios de los principales insumos de la industria (como el azúcar), cuyos incrementos no pueden ser trasladados al consumidor por la alta sensibilidad del precio respecto a la demanda. El gráfico 5 muestra la producción anual de bebidas gaseosas dulces y no dulces. ${ }^{7}$

7 Cabe mencionar que el volumen de producción de bebidas gaseosas sin dulce (light) se ha mantenido constante en los últimos años y, en términos de proporción, no resulta sig- 
Gráfico 5

Producción anual de bebidas gaseosas (millones de litros)

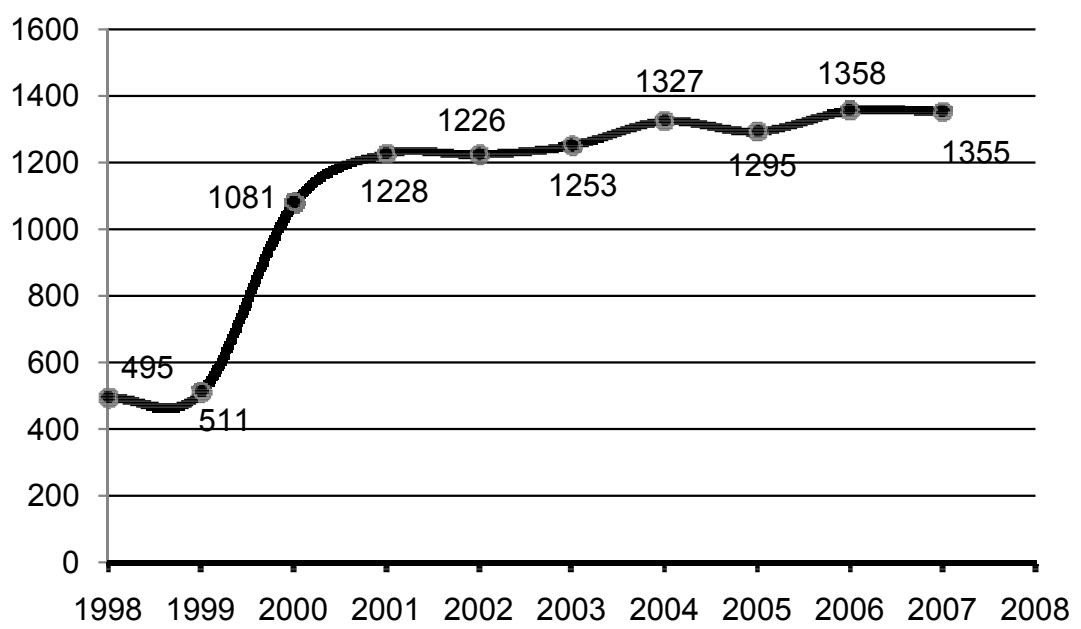

Fuente: Ministerio de la Producción (Produce) / Instituto Cuánto.

El consumo per cápita de bebidas gaseosas en el Perú es inferior a los 50 litros anuales por persona. Este promedio se ha mantenido prácticamente constante en los últimos años y está muy por debajo del promedio latinoamericano, que bordea los 70 litros. México tiene un consumo per cápita de 120 litros, Chile 90 litros y Argentina 70 litros. ${ }^{8}$ En provincias, el ratio resulta aún más bajo, ya que aproximadamente el $42 \%$ del consumo de gaseosas está concentrado en Lima. El gráfico 6 muestra la evolución del consumo per cápita de gaseosas en nuestro país.

nificativa respecto a los grandes volúmenes de gaseosas con dulce que produce el sector de nuestro interés.

8 Maximixe. CASER, Club de Análisis de Riesgos. Gaseosas. Marzo del 2008, p. 82. 


\section{Gráfico 6}

Consumo per cápita de bebidas gaseosas (litros anuales por persona)

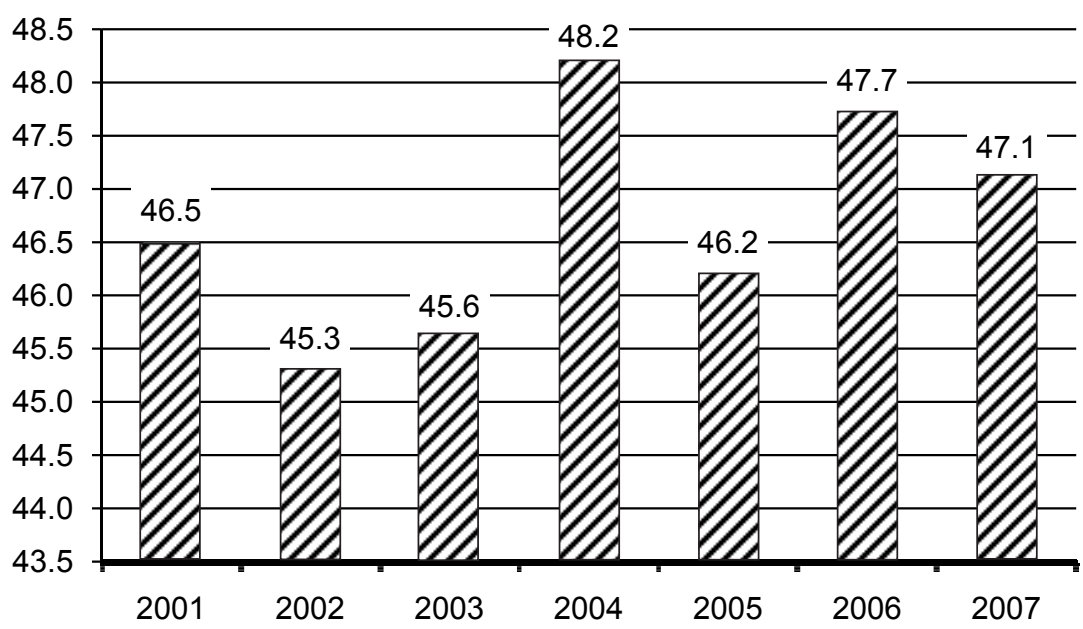

Fuente: Ministerio de la Producción, Sunat, INEI / Maximixe.

\section{EL PROCESO DE CONVERSIÓN DE BOTELLAS PET A FIBRA CORTA DE POLIÉSTER}

Enseguida detallaremos, por etapas, la propuesta de fabricación de fibra corta de poliéster a partir del scrap de botellas transparentes de PET. El scrap de botellas de PET se obtiene luego de proceder a la segregación de las botellas. Primero, estas son clasificadas por color, luego se les retiran tapas y etiquetas para, finalmente, ser molidas y obtener las hojuelas (scrap) de PET transparente.

Este insumo también se puede adquirir directamente a proveedores locales o, en su defecto, incluir su producción en el propio proceso de fabricación de la fibra corta de poliéster.

\subsection{Inspección}

El proceso de producción de la fibra corta de poliéster depende de una apropiada elección de la calidad del scrap de PET. Esto se logra con la adecuada selección del proveedor, quien debe ser capaz de suministrar un insumo con la calidad requerida. Sin embargo, para salvaguardar la calidad del producto final, es necesario empezar el proceso produc- 
tivo con la inspección y limpieza del material; evitando la presencia de residuos no plásticos, suciedad, restos metálicos, compuestos de papel o cartón, etcétera.

\subsection{Lavado}

El scrap de PET, libre ya de contaminantes, ${ }^{9}$ es lavado con agua a presión que contiene proporciones convenientes de detergente industrial para un proceso más efectivo. El material es enjuagado con agua pura y luego depositado en recipientes que tienen como base una malla metálica antioxidante de no más de $1 / 8$ de pulgada de diámetro, que permite que fluya el agua con los residuos aún presentes. Con ayuda de los recipientes, el scrap es luego transportado a la secadora.

\subsection{Secado}

Los fragmentos de PET, ya inspeccionados y limpios, antes de entrar al proceso de fundido para el hilado, deben ser secados bajo un constante control de temperatura. El secado puede ser al vacío (vaccum dryer) o, en su defecto, se emplea un sistema sencillo de flujo de aire caliente, gracias a resistencias eléctricas, suministrado por un ventilador. Posteriormente, el scrap ya libre de cualquier vestigio de humedad, puede continuar con el proceso siguiente.

\subsection{Fundido, filtrado y extrusión para hilatura}

Todo proceso de hilatura de fibra artificial se basa en tres etapas generales. Primero, la preparación de una solución viscosa (tipo jarabe). Segundo, la extrusión de esta solución a través de una tobera para formar la fibra. Finalmente, la solidificación de la fibra por coagulación, evaporación o enfriamiento.

9 Hay que cuidar la no presencia de PVC, nocivo para el funcionamiento de la maquinaria. Si el PET se contamina con PVC, su valor comercial disminuirá drásticamente. Incluso, dependiendo de su nivel de contaminación, puede quedar inservible. Tampoco debe haber presencia de otros polímeros, que alterarían el proceso por sus diferentes puntos de fusión (PP, HDPE, LDPE, PPS, etcétera). 
El scrap de PET se constituye en solución al fundirlo. ${ }^{10}$ Esta solución se conoce como solución de hilatura o pasta hilable. La solución se filtra antes de ser extruida.

La extrusión es una parte muy importante del proceso de hilatura. Consiste en forzar o bombear la solución de hilatura a través de los pequeños orificios de una hilera o tobera. Una hilera es una boquilla pequeña, semejante a un dedal. Las fibras obtenidas por la extrusión se enfrían y endurecen al hacer contacto con el aire. Para elaborar una mecha se recolectan fibras de varias hileras. Cada mecha contiene unos 2.400 den. ${ }^{11}$ Las mechas se unen para formar el sub-tow que se coloca en recipientes (canecas) capaces de almacenar hasta 300 kilogramos. ${ }^{12}$ Luego, las canecas se colocan en filetas, ${ }^{13}$ esperando la siguiente etapa del proceso.

\subsection{Estiramiento}

Previo baño en una emulsión de agua y aceite, el sub-tow debe ser estirado. Las fibras artificiales, al ser extruidas, presentan un estado molecular aleatorio, sin orientar. El estirado o alargamiento aumenta la cristalinidad y distribución interna ordenada, reduce el diámetro (disminuyendo por consiguiente el título o denier) y agrupa las moléculas juntándolas más. La cristalinidad y orientación se relaciona con propiedades físicas de la fibra. La resistencia a la abrasión, la elongación, la absorción de humedad, así como la receptividad de la fibra a los colorantes, son algunas de esas propiedades.

Los poliésteres deben estirarse en caliente para que la alineación molecular sea efectiva. Las cadenas moleculares se mantienen unidas entre sí por enlaces cruzados o por fuerzas intermoleculares (llamadas enlaces de hidrógeno y fuerzas de Van der Waals). Las fuerzas son similares a la atracción entre un imán y un trozo de hierro. Mientras más cerca estén las cadenas unas de otras, más fuertes serán los enla-

$10 \mathrm{El}$ punto de fusión del PET está entre los $250^{\circ} \mathrm{C}$ y $260^{\circ} \mathrm{C}$.

11 El denier (den) es un indicador que relaciona el peso y la longitud del filamento. Un denier (1 den) significa que 9.000 metros de ese filamento pesan un gramo (1g).

12 Un grupo de mechas formarán el llamado sub-tow de 40.000 den.

13 Una fileta puede tener 24 canecas. Cada una contiene el llamado sub-tow. 
ces. El enlace de hidrógeno es la atracción de los átomos positivos de hidrógeno en una cadena por átomos negativos de oxígeno o nitrógeno de una cadena continua. Las fuerzas de Van der Waals son similares, pero más débiles. ${ }^{14}$

\subsection{Rizado (crimpado) y secado}

El rizado de la fibra se refiere a las ondas, quiebres, rizos o dobleces a lo largo de su longitud. Este tipo de ondulación aumenta la cohesión, resiliencia, resistencia a la abrasión, elasticidad, volumen y conservación del calor. El rizado también aumenta la absorbencia, y si bien favorece la comodidad al contacto con la piel, puede reducir el lustre. Una forma común de rizado es el mecánico, que se imparte a la fibra haciéndola pasar a través de rodillos gravados, torciéndolas o aplanando uno de sus lados.

El rizado o crimpado favorece la cohesión. Es decir, la capacidad de las fibras de permanecer juntas durante la hilatura. Además, contribuye a darle resistencia al deshilachado de la tela posteriormente. ${ }^{15}$

El sub-tow, una vez rizado, pasa por un túnel de secado para fijar el rizo en la fibra. La mecha continua se llama ahora tow, quedando lista para la obtención de fibra corta, dándole la longitud de corte deseada ( 15 den y 3 den para la mezclas con algodón y rayón, respectivamente). También el tow puede ser acondicionado para mezcla con lana si es cortado de manera especial para este fin (unos 6 den).

\subsection{Cortado y embalado}

Finalmente, el tow se corta en las longitudes predeterminadas y se embala en pacas de unos 300 kilogramos, ajustadas con zunchos de plástico. El producto final está listo para ser comercializado bajo el nombre de fibra corta de poliéster, de acuerdo a las especificaciones solicitadas y requeridas por el mercado.

A continuación se presenta el Diagrama de Operaciones del Proceso, a fin de resumir las etapas anteriormente descritas.

14 Hollen, Norma. Manual de los textiles, 1990, pp. 139-140.

15 Ibídem, p. 139. 


\section{Gráfico 7}

Diagrama de operaciones del proceso para la producción de fibra corta de poliéster

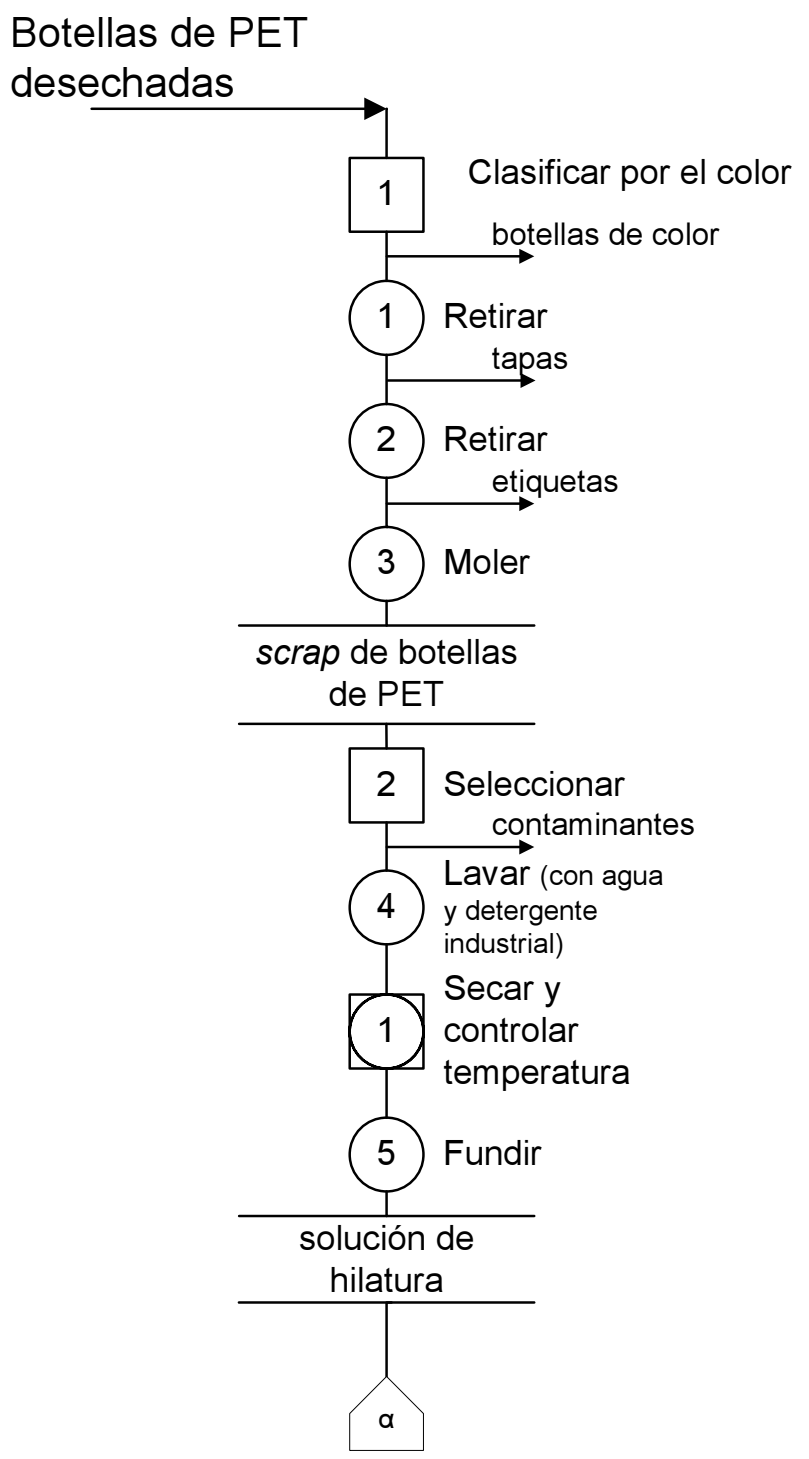

(continúa) 

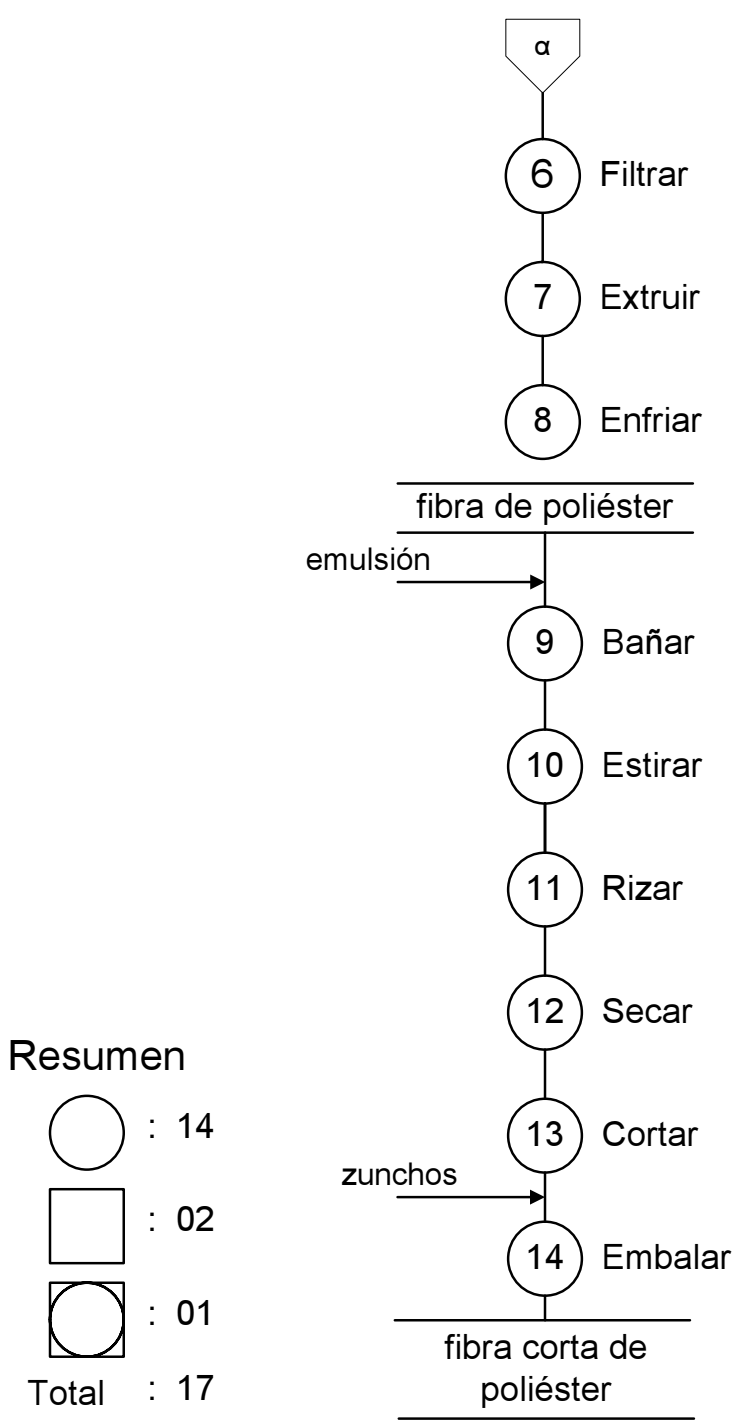

Elaboración propia. 


\section{LOS USOS DEL POLIÉSTER RECICLADO}

La necesidad de buscar alternativas de reutilización para algunos materiales, radica en el imperativo ético de preservar nuestro planeta, ya desgastado y afectado por la contaminación. Reciclar PET es reintegrar este polímero en un nuevo ciclo productivo como materia prima. Las botellas sin pigmento (transparentes) tienen mayor valor para el reciclado por sus sendas posibilidades de uso; entre otros, como material de empaque, láminas para termoformados, madera plástica, tarimas y fibra de poliéster.

La atención puesta en el presente artículo en la fibra corta de poliéster, radica en que es la fibra sintética de uso más extendido en la fabricación de tejidos de diferentes características, ya sea como componente único o mezclado con otras fibras naturales, como el algodón. Entre los productos fabricados a partir de esta fibra tenemos casacas, suéteres, frazadas, alfombras, telas para decoración (cortinas y tapicería), ropa de cama, tejidos anchos (toldos, lonas, carpas, cintas transportadoras, carteles luminosos, bolsos de viaje) y tejidos estrechos (cinturones de seguridad, cintas de transmisión, mangueras, etcétera).

Finalmente, nuestra propuesta tiene por finalidad divulgar y sensibilizar a los interesados en la tecnología industrial del reciclaje, en torno a una de las tantas posibilidades de reutilización del PET, en una apuesta universal por tecnologías limpias que contribuyan a paliar en algo el inmenso daño ambiental que en las últimas décadas hemos generado. 


\section{BIBLIOGRAFÍA}

Aprepet, A. C. [en línea]. <http://www.aprepet.org.mx>. [Consulta: mayo del 2009.]

Brilén, S.A. Empresa productora de hilo de poliéster [en línea]. $<$ http://www.brilen.com/index.jsp>. [Consulta: mayo del 2009.]

Cuánto. Anuario Estadístico Perú en Números. Lima: Instituto Cuánto, octubre del 2008.

Hollen, Norma. Manual de los textiles. . $^{\mathrm{a}}$ edición. México, D.F.: Ediciones Ciencia y Técnica S.A., 1990.

Maximixe. CASER, Club de Análisis de Riesgos. Gaseosas. Lima: Maximice Consult S.A. Marzo del 2008.

—_. Agua embotellada. Lima: Maximice Consult S.A. Abril del 2008. Envases plásticos. Lima: Maximice Consult S.A. Febrero del 2009.

Plastivida Argentina [en línea]. <www.plastividad.com.ar>. [Consulta: mayo del 2009.]

Ruiz Ruiz, Marcos. "Estudio de prefactibilidad para la elaboración de fibra de poliéster a partir de botellas desechadas de bebidas gaseosas". Tesis para optar el título de ingeniero industrial. Lima: Universidad de Lima, 2001. 